\title{
El sentido social del profesionalismo médico
}

\author{
Nina Horwitz Camposa \\ The social meaning \\ of medical professionalism
}

This essay looks into the meaning of today's professionalism and the apparent inconsistency between its growing status and a context of profound changes and dissatisfaction with medical practice. The cultural climate of our times is reviewed, as the framework for understanding changes in the social organization of medicine. One of the critical traits of professions has been their power to manage specialized knowledge under their own standards, without external control. The limits of this self regulation of expertise have faded, representing an important threat for professionalism. Together with the fundamental changes and tensions of work conditions for professionals, however, the appeal of professionalism is on the rise. Because of its ability to submit devastating individualism to collective interests, professionalism can contribute to the stability of social systems, as a third logic, between the market and public organizations. In medicine, professionalism as a value based system and ideology, also emerges as a response to the challenges posed to its practice. It represents reflection and preservation of traditional values inspiring a practice of excellence, which will undoubtedly restore and enhance public confidence in medicine (Rev Méd Chile 2006; 134: 520-4).

(Key words: Ethics, medical; Practice management, medical; Professionalism; Sociology)

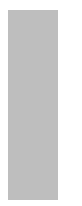

Recibido el 25 de julio, 2005. Aceptado el 11 de agosto, 2005.

Trabajo basado en la ponencia homónima presentada en el panel sobre Profesionalismo, en el XXVI Congreso de Medicina Interna, Viña del Mar, octubre de 2004.

Departamento de Bioética y Humanidades Médicas y Departamento de Psiquiatría y Salud Mental Oriente, Facultad de Medicina, Universidad de Chile. Santiago de Chile.

aSocióloga

\begin{abstract}
-Cuál es el significado que se le asigna al Concepto de profesionalismo hoy? ¿Cómo se explica el auge del profesionalismo en nuestros días? Para responder a esas interrogantes, se abordarán tres temas: sobre los cambios en el contexto social y cultural de la práctica médica y los conceptos de profesión y de profesionalismo.
\end{abstract}

\section{EL CAMBIO EN LA PRÁCTICA MÉDICA}

La práctica de la profesión médica ha cambiado y tiene poco que ver con la de hace sólo algunas décadas. Un distinguido ensayista afirma que, con la globalización, se ha instalado en nuestras vidas "la sensación de que nuestro empleo, comunidad o lugar de trabajo pueden verse modificados, en cualquier momento, por fuerzas económicas y tecnológicas anónimas que distan mucho de ser estables" ${ }^{\prime 1}$. Estas tensiones constituyen un desafío para el concepto mismo de profesión ${ }^{2}$.

Los múltiples análisis de la sociedad occidental contemporánea, han enfatizado diversos aspectos de ella, al caracterizarla. Entre éstos, algunos ofrecen una clara visión del "clima de época", relevante al ejercicio de la medicina. 
En primer lugar, la noción de sociedad de la modernidad tardía, caracterizada por un creciente ejercicio reflexivo. La mentalidad moderna, forjada por la ciencia y el pensamiento racional de la Europa de la Ilustración, se ha intensificado y generalizado, con lo cual una proporción mayoritaria de las dimensiones de la vida social y de las relaciones materiales con el ambiente natural, se hacen susceptibles a una constante revisión, a la luz de la renovación del conocimiento y de la información. Se ha institucionalizado la duda radical, instigada por el planteamiento de que todo conocimiento es tentativo y perfectible, lo que ha surgido, en la primera mitad del siglo veinte, de la idea de ciencia como un conjunto de enunciados hipotéticos contrastables ${ }^{3}$. Esta noción otorga a la inducción científica la cualidad de instancia justificadora de la verdad, pero le quita el carácter de objetividad absoluta y universal. Hoy, la relación entre la medicina y la población se construye alrededor de un proceso dialéctico, entre una necesaria confianza y la duda, confianza activa que ahora debe ser ganada y retenida, considerando una situación de incertidumbre creciente, que es la propia del ejercicio médico.

La sociedad del riesgo ${ }^{4}$, en que las dimensiones globales de los riesgos han llegado a constituir un desafío para la reflexión ética y el control social responsable, que pueden ofrecer una guía de protección y tranquilidad a la actuación individual, en las profesiones tecnológicas.

La sociedad de la información, que pone sobre el tapete la disponibilidad de información especializada para todos. Tal mediación de la experiencia es particularmente sensible en la medicina, reflejando una actitud colectiva ambivalente y polarizada.

La sociedad de individuos ${ }^{5}$, que plantea al profesional el auge de los valores del individualismo con una fluida movilidad, pero acompañada al mismo tiempo de inestabilidad e incertidumbre en las condiciones de empleo, se traduce también en la necesidad de proporcionar anclajes simbólicos, de referencia e identidad, a un sujeto desorientado.

La sociedad burocrática o corporativa $^{5}$, que subraya la presencia de las organizaciones en la vida laboral moderna y su intensa burocratización. Los médicos se verán obligados a proyectar su ejercicio profesional, casi exclusivamente en el marco de organizaciones en las cuales detenten la calidad de funcionarios públicos o de corporaciones privadas ${ }^{2}$.

El segundo hecho a constatar es el de una atmósfera de insatisfacción generalizada con la medicina, que afecta a una población crecientemente crítica $\mathrm{y}$, también, a los médicos. Los cambios en el ejercicio de la profesión han tenido un fuerte impacto en el bienestar subjetivo de los médicos. Muchos de ellos, están viviendo situaciones de malestar, frustración y desencanto con la profesión que han sido profusamente comunicadas, últimamente, en publicaciones internaciona$\operatorname{les}^{6-8}$ y nacionales ${ }^{2,9,10}$. Pareciera ser un hecho universal que los médicos están teniendo que adaptarse a un ambiente laboral nuevo y extraño.

En el contexto descrito, se plantea la cuestión del profesionalismo en nuestros días. Pareciera que nos enfrentamos a una paradoja: mientras, por una parte, se discute acerca de la importancia del profesionalismo en la práctica médica y sus características, por otra, muchos médicos sufren los embates de la desilusión con la profesión, como precio del cambio y el progreso. ¿Cómo explicar esta contradicción? ¿Será más aparente que real y, la medicina, puede continuar siendo la profesión paradigmática que ha sido?

En el rol social del médico se integran dos dimensiones, la de sanador y la de profesional ${ }^{11}$. En el mundo occidental, la tradición de sanar o curar, se remonta a la Grecia helénica y al juramento de Hipócrates. Las profesiones, por su parte, se originan en los gremios y universidades de la Europa medieval. Sin embargo, su sentido actual lo adquieren en la modemidad, en que se separan de la esfera religiosa, carácter que se refuerza con la industrialización y la división del trabajo. Ambas dimensiones, se vinculan por códigos éticos que gobieman el comportamiento de sus miembros y por las ciencias naturales y humanistas que las respaldan. Resulta interesante tener presente estas dos facetas del rol médico, ya que lo que hoy resulta más cuestionado por la población, es el prestigio colectivo de la profesión, más que la relación clínica, que sigue siendo percibida por los pacientes, en general, en forma favorable.

\section{EL CONCEPTO DE PROFESIÓN}

Antes de contribuir a la discusión sobre el concepto de profesionalismo, es necesario referirse al de 
profesión. Para estos propósitos, cabe recordar las características esenciales de una profesión ${ }^{5}$ :

- Dominio de un cuerpo complejo de conocimientos y habilidades específicas, adquirido luego de un período largo de formación.

- Control, con capacidad normativa, sobre el trabajo basado en aquel conocimiento.

- Dispone de organizaciones profesionales reconocidas legalmente, con capacidad reguladora del acceso a la profesión y todo lo concerniente a su práctica.

- Posee un código ético y tiene la potestad exclusiva para aplicarlo. Todo el grupo profesional ha de profesar un conjunto de valores que muchos autores coinciden en resumir en: altruismo, integridad, disciplina, eficiencia $\mathrm{y}$ compromiso.

El ejercicio de la profesión no es sólo una actividad individual. Ella es desempeñada por un conjunto de "colegas" (collegium), en el sentido amplio del término, quienes asumen su ethos o carácter, formando una comunidad, que les brinda identidad y pertenencia. Desde el punto de vista ético, la actividad profesional no es sólo un medio para conseguir una meta que está situada fuera de ella (el ingreso). La meta que le da sentido y legitimidad social y moral es interna, y consiste en proporcionar a la sociedad un bien específico ${ }^{12}$. En el caso de la medicina, ese fin es la salud de los pacientes.

Centrándose paradigmáticamente en la medicina, el sociólogo Eliot Freidson, marcó un hito en el estudio de las profesiones, hace más de 20 años, destacando que, en contraste con otro tipo de ocupaciones, tendrían la capacidad para acumular y usar conocimientos bajo sus propios estándares, sin estar sujetos a control por actores externos $^{13}$. Hoy son precisamente los perfiles de esa autonomía los que se han tornado más borrosos, y su menoscabo constituye uno de los factores predominantes de amenaza al profesionalismo. Las principales críticas que han puesto en jaque el status profesional de la medicina provienen de la desconfianza, a la cual contribuyen principalmente dos factores: la percepción de que la profesión no ha logrado autorregularse para garantizar la calidad de la práctica y que muchos profesionales han puesto su propio interés por sobre el de los pacientes y la sociedad.
DEL CONCEPTO DE PROFESIÓN A LAS CONSIDERACIONES SOBRE EL PROFESIONALISMO

Junto a las tensiones y los cambios fundamentales en el entorno en que los profesionales trabajan hoy, se ha ido despertando el interés y generando consenso acerca del atractivo de la noción de profesionalismo en diferentes contextos laborales, no solamente en la medicina. Por ejemplo, se utiliza como un mecanismo de mercado para atraer clientes y en los planteamientos sobre misión y metas organizacionales, para motivar a los empleados o funcionarios. Del mismo modo, calificamos un trabajo de calidad, como "profesional". Éstos y otros indicadores sociales, nos sugieren la necesidad de un cambio de foco, desde una preocupación por la "profesión" a un análisis sobre el "profesionalismo", como motivador y facilitador de cambio y renovación ocupacional ${ }^{14}$.

Las variadas interpretaciones del concepto de profesionalismo, en la literatura de las ciencias sociales, pueden agruparse con fines analíticos en dos grandes categorías: como sistema normativo de valores, por una parte, y como ideología de control, por otra. La diferencia más radical entre ambas, es que mientras el profesionalismo, como sistema de valores, se muestra optimista frente a una contribución positiva del concepto a la mantención de un orden social regulado, el profesionalismo como ideología, destaca su carácter de sistema hegemónico de principios y como medio de control de la conducta profesional.

Desde hace mucho tiempo, han existido intentos por analizar el sentido y las funciones del profesionalismo, para la estabilidad y equilibrio de los sistemas sociales. Durkheim planteó, hace casi un siglo, el profesionalismo como una suerte de comunidad moral, basada en la pertenencia ocupacional $^{15}$. Otros autores, posteriormente, han percibido al profesionalismo como capaz de someter el individualismo avasallador a intereses colectivos, como defensa de los procesos democráticos estables, o han enaltecido sus características de altruismo y orientación de servicio ${ }^{14}$. El profesionalismo, en su esencia, implica reconocer la importancia de la confianza en las relaciones sociales y económicas, en las sociedades modernas, con una intensa división del trabajo; los legos deben depositar su confianza en los profesionales. Pero el profesionalismo, también, requiere que los 
profesionales sean merecedores de esa confianza, en virtud de la cual serán recompensados con autoridad, privilegios y mayor status.

En las décadas de los 70 y 80, el concepto de profesionalismo como sistema de valores, fue reemplazado por evaluaciones críticas de las profesiones, como grupos monopólicos poderosos, privilegiados y centrados en sus propios intereses ${ }^{14}$. Gradualmente, sin embargo, la visión del profesionalismo como ideología fue demostrando flaquezas. Así como en algunos momentos las profesiones ejercen su poder como actores sociales o grupos de interés, es más frecuente que ellas tengan que responder a demandas externas, que pueden ser políticas, económicas, culturales o sociales. Algunos autores han investigado ciertos aspectos específicos de la hegemonía profesional de los médicos, pero inevitablemente ligada a otros procesos, como la fragmentación, la estratificación y la creciente jerarquización interna de la profesión misma. Sólo algunos médicos, y no todos, consiguen poder y privilegios sociales, por ejemplo. Esto ha renovado el interés en el profesionalismo como sistema valórico, recogiendo la evidencia histórica acerca del proceso paralelo de creación de los estados-nacionales modemos y de las profesiones, como expresión de principios liberales. Recientemente, se ha profundizado esta línea argumental al considerar la necesidad de un orden social pluralista en las economías globales y los mercados internacionales, y la contribución de las organizaciones profesionales a esta aspiración, con un proyecto valórico y normativo.

En este marco, Freidson, ha planteado en dos publicaciones recientes ${ }^{16,17}$, que el profesionalismo constituye una forma única de regulación del ejercicio profesional, con claras ventajas sobre el mercado y el estado. Analizando las fuerzas sociales, políticas y económicas que ejercen presión hoy sobre las profesiones, el autor plantea que los métodos del libre mercado o burocráticos y organizacionales, empobrecen y estandarizan la calidad del servicio a la población, y desmotivan a los profesionales que lo entregan, agregando que esto puede ser neutralizado reforzando las virtudes del profesionalismo. El profesionalismo se presenta como una forma de organización social, con su propia racionalidad interna, que representa una tercera lógica, frente al mercado y al estado.

En la medicina, el profesionalismo, como sistema valórico y como ideología de protección de su práctica, se perfila hoy como una respuesta para superar los desafíos planteados a su ejercicio. Para ello, no basta sólo con mejores condiciones estructurales, materiales y económicas, en el ejercicio de la práctica médica. El profesionalismo, constituye un esfuerzo por rearticular y reenfatizar los valores que han caracterizado, tradicionalmente, a la medicina. Desde el punto de vista social, representa una oportunidad que descansa en un posicionamiento activo para reafirmar los valores del ideario profesional, para promoverlos, fomentarlos y ejercerlos. Sobre esta base, y con la vista puesta en el futuro, podrá renovar el pacto 0 contrato con la sociedad, necesidad a la que se ha hecho reiterada referencia en la literatura, ${ }^{8,18-22}$. De aquí deriva el imperativo para que los médicos comprendan el origen y la naturaleza del status profesional y de las obligaciones para sustentarlo. El profesionalismo puede y debe enseñarse, y ser evaluado, bajo la responsabilidad de los organismos docentes y acreditadores de la práctica médica. Del mismo modo, le cabe en esto una responsabilidad a la sociedad; en el estímulo y apoyo, a través de las estructuras del sistema de salud, que permitan el ejercicio y la preservación de los valores éticos en los médicos.

Por la paradoja planteada inicialmente, la pregunta es, entonces, más aparente que real. El profesionalismo representa un camino para sobreponerse al malestar e insatisfacción en el ejercicio individual. La importancia del profesionalismo desde un punto de vista socio antropológico, además de lo expuesto, radica en sus implicaciones como forma de organización social. Su origen como movimiento, al interior de la profesión médica, constituye, como ya se señaló, una tercera lógica o una tercera vía ante las burocracias públicas y el mercado. Expresa, además, el poder de la acción de la sociedad civil para adaptarse, constructivamente, a los cambios y responder a los desafíos psicosociales de las profesiones. Un indicador de este movimiento se aprecia en la vasta literatura sobre las profesiones, que hasta hace poco tiempo, se encontraba casi exclusivamente en las ciencias sociales y la filosofía. Esto no siempre fue afortunado porque ellas han sido, en ocasiones, muy críticas de la profesión médica, lo cual reflejaba $\mathrm{y}$, a su vez, contribuía a condicionar la opinión pública y las políticas de salud. Los médicos no estaban entera- 
mente conscientes de su efecto en la percepción social de la profesión. Este panorama ha cambiado drásticamente en la última década, en que se aprecian extensos y lúcidos análisis en publicaciones y reuniones médicas, que traducen inquietud pero también reflexión y asentamiento de valores tradicionales relevantes en el ejercicio de la

\section{REFERENCIAS}

1. Friedman TL. The Lexus and the Olive Tree: Understanding Globalization. New York: Anchor Books, 2000.

2. Horwitz N. El cambio de la práctica médica. Desafíos psicosociales para la profesión. Rev Méd Chile 2004; 132: 768-72.

3. Hempel C. Filosofía de la ciencia natural. Alianza, 1979.

4. Веск U. La sociedad del riesgo: hacia una nueva modernidad. Barcelona, Paidós, 1986.

5. Pardell H. ¿Tiene sentido hablar de profesionalismo hoy? Educación Médica 2003; 6: 63-80.

6. KASSIRER JP. Doctor Discontent. N Engl JM 1998; 339: 1543-5.

7. Sмтtн R. Why are Doctors so Unhappy? BMJ 2001; 322: 1073-4.

8. Edwards N, Kornacki MJ, Silversin J. Unhappy Doctors: What are the Causes and What can be Done? BMJ 2002; 324: 835-6.

9. JiMÉNEZ JP. Bienestar y satisfacción de los médicos con el ejercicio de su profesión en un sistema de salud en un proceso de reforma. Rev Méd Chile 2004; 132: 635-6.

10. JIMÉNEZ JP. Cambios en el entorno del trabajo médico. Rev Méd Chile 2004; 132: 737-42.

11. Cruess SR, Johnston S, Cruess RL. Professionalism for Medicine: Opportunities and Obligations. MJA 2002; 177: 208-11.

12. Cortina A. El sentido de las profesiones. En Cortina A y Conill J. 10 palabras claves en la ética de las profesiones. Navarra, España: Editorial Verbo Divino, 2000. medicina, que son expresión de excelencia profesional $^{23,24}$. Ello permite, también, prever un aumento mayor y paulatino y la restauración de la confiabilidad de los usuarios en la calidad de la medicina, en su sentido más amplio, como servicio dispuesto a sus demandas en salud ${ }^{24,25}$.

13. FreIDSON E. La profesión médica. Un estudio de sociología del conocimiento aplicado. Barcelona: Península, 1978.

14. EvETTS J. The Sociological Analysis of Professionalism. Occupational Change in the Modern World. International Sociology 2003; 18: 395-415. SAGE.

15. DuRKHeIM E. Professional Ethics and Civil Morals. London: Routledge, 1992.

16. FREIDSOn E. Professionalism Reborn: Theory, Prophecy and Policy. Cambridge: Polity, 1994.

17. Freidson E. Professionalism: The Third Logic. London: Polity, 2000.

18. LUDMERER KM. Instilling professionalism in medical education. JAMA 1999; 282: 881-2.

19. Wynia MK, Latham SR, Kao AC, Berg JW, Emanuel $\mathrm{L}$. Medical professionalism in society. N Engl J Med 1999; 341: 1612-6.

20. Swick HM. Towards a normative definition of medical professionalism. Acad Med 2000; 75: 612-6.

21. EPSTEIN RM, HundeRT EM. Defining and assessing professional competence. JAMA 2002; 287: 226-35.

22. EFIM, ACP-ASIM, ABIM. Profesionalismo médico en el nuevo milenio: un estatuto para el ejercicio de la medicina. Rev Méd Chile 2003; 131: 457-60.

23. STERN DT. Practicing what we preach? An analysis of the curriculum of values in medical education. Am J Med 1998; 104: 569-75.

24. Cruess RL, CRUess SR. Teaching medicine as a profession in the service of healing. Acad Med 1997; 72: 941-52.

25. Rosselot E. Aseguramiento de la Calidad Profesional. Un nuevo marco ético para el ejercicio de la medicina. Rev Méd Chile 1999; 127: 1381-9. 